\title{
Efficacy-specific herbal group detection from traditional Chinese medicine prescriptions via hierarchical attentive neural network model
}

\author{
Li Chen ${ }^{1 *+} \mathbb{D}$, Xinglong Liu ${ }^{2+}$, Siyuan Zhang ${ }^{1}$, Hong Yi ${ }^{1}$, Yongmei Lu ${ }^{1}$ and Pan Yao ${ }^{1}$
}

\begin{abstract}
Background: Mining massive prescriptions in Traditional Chinese Medicine (TCM) accumulated in the lengthy period of several thousand years to discover essential herbal groups for distinct efficacies is of significance for TCM modernization, thus starting to draw attentions recently. However, most existing methods for the task treat herbs with different surface forms orthogonally and determine efficacy-specific herbal groups based on the raw frequencies an herbal group occur in a collection of prescriptions. Such methods entirely overlook the fact that prescriptions in TCM are formed empirically by different people at different historical stages, and thus full of herbs with different surface forms expressing the same material, or even noisy and redundant herbs.
\end{abstract}

Methods: We propose a two-stage approach for efficacy-specific herbal group detection from prescriptions in TCM. For the first stage we devise a hierarchical attentive neural network model to capture essential herbs in a prescription for its efficacy, where herbs are encoded with dense real-valued vectors learned automatically to identify their differences on the semantical level. For the second stage, frequent patterns are mined to discover essential herbal groups for an efficacy from distilled prescriptions obtained in the first stage.

Results: We verify the effectiveness of our proposed approach from two aspects, the first one is the ability of the hierarchical attentive neural network model to distill a prescription, and the second one is the accuracy in discovering efficacy-specific herbal groups.

Conclusion: The experimental results demonstrate that the hierarchical attentive neural network model is capable to capture herbs in a prescription essential to its efficacy, and the distilled prescriptions significantly could improve the performance of efficacy-specific herbal group detection.

Keywords: Efficacy-specific herbal group, Hierarchical attentive neural network, Traditional Chinese medicine, Prescription

\section{Background}

Traditional Chinese Medicine (TCM) has a long history of several thousand years, and during such long period massive prescriptions are accumulated. These prescriptions were providing effective protection for ancestors

*Correspondence: cl@scu.edu.cn

${ }^{\dagger}$ Li Chen and Xinglong Liu are equally contributed to these work.

${ }^{1}$ Department of Computer Science, Sichuan University, Chengdu, China

Full list of author information is available at the end of the article of Chinese people from different diseases for thousands of years, and are applied widely even nowadays [1]. However, the prescriptions are formed by different people at different historical times empirically or with trial and error, without agreed and rigorous theoretical support or specification, which results in an uneven quality in these prescriptions and further hinders them from more extended applications in modern society. In order to refine these massive prescriptions and discover 
valuable patterns of herbal usages in TCM behind the prescriptions, data mining and machine learning technologies are incorporated in recent years $[2,3]$ such as association rule mining to discover herbal groups for alopecia treatment [4] and for syndrome differentiation of TCM [5], random walk and label transmission to detect herbal groups [6], multi-content Latent dirichlet allocation(LDA) to recommend prescriptions for the patients of amenorrhea and lung cancer [7], and supervised learning to perform classification for TCM clinical records [8]. Among all the issues tried with data mining and machine learning technologies, detecting essential herbal groups for a particular efficacy is crucial for the science of TCM formulas as well as for the practical functionality of the TCM prescriptions in modern society $[6,9]$

The efficacy of a prescription is not simply the addition of all herbs. On the contrary, they interact with each other and show better curative efficacy and fewer side effects than a single one. Besides, the efficacy of a single herb generally is diverse, and practitioners need to control single herbs with multi-efficacy to play an expected efficacy during diagnosing disease and making prescriptions. For example, the efficacies of Rhizoma Rhei(大黄 ) are heat-clearing and detoxicating, removing accumulation with purgation, and so on. When combined with Coptis chinensis (黄连) and Radix Scutellariae(黄芩), Rhizoma Rhei can deliver the efficacy of heat-clearing and detoxicating. On the other hand, Rhizoma Rhei plays the efficacy of removing accumulation with purgation when combined with mirabilite (芒硝). Hence, TCM doctors not only know the efficacy of herbs but also master the efficacy of herbal groups when making prescriptions.

Therefore, TCM researchers draw more and more attentions recently in herbal group mining, and gain some promising results [10-13]. However, most existing methods for the task determine essential herbal groups based only on the raw frequencies with which herbal groups occur in a collection of prescriptions. Although other variations to these raw-frequency-based methods exist, such as the one using random walk technique on a herb graph based on a similarity measurement between herbs with their features $[6,14]$, in fact they are all heuristic and feature-engineering-based, treating herbs with different surface forms orthogonally and overlooking entirely the fact that prescriptions in TCM are formed empirically by different people at different historical times, and thus full of herbs with different surface forms expressing the same material, or even noisy and redundant herbs.

In order to overcome the difficulties mentioned above, in this paper we devise a two-stage approach to detect efficacy-specific herbal groups (ESHGs) from prescriptions in TCM. The first stage is performing recognition of essential herbs in a prescription for its efficacy, and the second stage collects all prescriptions of an efficacy with their respective essential herbs to discover ESHGs. For the first stage a hierarchical attentive neural network model is employed, and by means of its attention mechanism, essential herbs in a prescription for its efficacy are recognized. For the second stage frequent pattern mining is incorporated to discover the ESHGs of an efficacy from its distilled prescriptions obtained in the first stage. We conduct a series of experiments to verify the effectiveness of our two-stage approach from two angles. The first one is the ability of the attention mechanism to capture essential herbs for a prescription efficacy and thus distill the prescription for subsequent frequentpattern-based ESHGs detection, and the second one is the quality of the ESHGs discovered with our two-stage approach. The experimental results demonstrate that the hierarchical attentive neural network model is capable to capture herbs in a prescription essential to its efficacy, and the distilled prescriptions significantly improve the effect of ESHGs detection.

The major contributions of this work are three-fold: (1) a deep learning model is incorporated into the ESHGs detection task, where a dense real embedding is learned for every herb to capture semantical correlations among herbs, thus overcoming enormous differences in herbal name usages in the long period of TCM history; (2) a hierarchical attentive mechanism is proposed to capture essential herbs in a prescription for its efficacy to distill the prescription for the purpose of improving ESHGs detection; (3) a series of experiments is conducted to investigate the performance of our model for identifying herbs in a prescription essential to its efficacy and detecting ESHGs for a particular efficacy, and the experimental results verify the effectiveness of our two-stage approach.

\section{Related work}

In recent years, many disciplines witness rapid growth in employing data-intensive approaches based on machine learning and data mining technologies to discover patterns hidden in a massive volume of data. Likewise, some efforts have emerged in TCM recently which utilize machine learning and data mining technologies for discovering knowledge from TCM literature [15-18], clinical records $[8,19,20]$, and prescriptions [12, 13, 21-26]. Among all these data mining tasks the last one draws particular attention due to the fact that, prescriptions as the primary knowledge sources for TCM are invented mostly with empirical experiences in a long historical span of times and distilling knowledge from them are far from completion, which hinders applications of TCM in modern society. 
Data mining and machine learning from prescriptions are hopeful to cover theoretical gaps in TCM and lift practical performance in health care and disease treatment.

With regard to types of knowledge to be discovered from prescriptions in TCM, herbal groups having a particular efficacy are of great significance for TCM theory as well as for its practice, thus becoming a primary focus in recent works on TCM prescription data mining and knowledge discovery. The efficacy of herb has been identified and summarized through serval thousand years of clinical practices, but there are many herbal groups for disease/symptom/efficacy still needed to be further mined. Current approaches to perform such task proposed by now are mostly utilizing co-occurrence frequency of herbs in a prescription collection to measure their strengths in forming a herbal group with a particular efficacy or toward a particular disease/symptom. While initially the Apriori algorithm or its some accelerated variations are employed in these frequency-based approaches $[10-13,18,27]$ due to their simplicity and ease of implementation, recently Han et al. [13] proposed to mine frequent patterns not only in occurrence of herbs, but also in their absence in a collection of prescriptions. The purpose of the latter is to discover simultaneously collocated as well as contraindicated combinations of herbs for a particular disease, thus potentially having more applications. However, the above proposed approaches, including the one in [13], are all lacking in empirical evaluation for their quality of the generated herbal groups, and the only evaluation reported in their original papers are efficiency in terms of the time expended for the mining. Furthermore, the rawfrequency-based approaches treat different herbs equally, ignoring skew distribution in their usage frequencies and their special properties, which obviously should result in poor quality herbal groups.

In order to overcome the difficulties faced by raw-frequency-based approaches, Wang et al. [6] incorporated features of herbs into the mining process, such as their nature and flavor. They measure the similarity for a pair of herbs according to their features and then construct a weighted undirected graph with the vertexes denoting herbs and the edges weighted with the corresponding similarity, and then a random walk processing is performed on the graph to discover required herbal groups. Yao et al. [21] proposed an LDA-based topic model to capture latent syndromes and herbal roles (i.e. jun-chen$z u o-s h i)$ in a prescription, thus a group of herbs for a set of symptoms can be inferred by the model.

Although the recent studies such [6] and [21] have made significant progress in TCM prescription mining, almost all of them treat the herbs orthogonally, representing them with one-hot manner. Such manner has to be faced with the difficulties of data sparsity. Furthermore, the one-hot manner in essence ignores possible correlations on the level of the representation, which is necessary for TCM herbs. Prescriptions of TCM are formed by different people at different historical times empirically in a way of trial and error, without agreed and rigorous theoretical support or specification. The forming process has resulted directly in an uneven quality in the prescriptions being full of herbs with different surface forms expressing the same material, and also the same surface forms denoting distinct herbs. Without distilling such formed prescriptions, the raw-frequency-based and one-hot-based approaches are difficult to achieve satisfying prescription mining results.

To tackle the issues mentioned above, a two-stage approach to detect essential groups for a particular efficacy from TCM prescriptions is proposed in this paper. In the first stage, we devise a neural network model to distill prescriptions in TCM, in which herbs are embedded into dense real vectors learned automatically to capture the semantical correlations, and a hierarchical attentive mechanism is proposed to recognize essential herbs in a prescription for its efficacy, filtering out insignificant herbs. For the second stage, ESHGs are mined from the distilled prescriptions acquired in the first stage. Finally, we collect a set of efficacies labeled with their known herbal groups by TCM experts as the gold-standard test data to verify the effectiveness of our approach in discovering ESHGs.

\section{Methods}

As discussed above, our solution to ESHGs detection consists in a two-stage approach, in which the first stage is to capture essential herbs in a prescription for its efficacy thus resulting in a cleaned collection of prescriptions, and the second stage is to discover ESHGs from the distilled prescriptions. The architectural diagram is given in Fig. 1 and will be detailed below in this section.

In order to capture essential herbs in a prescription for its efficacy, we devise a hierarchical attentive neural network with its sigmoid output, denoting the probability of a prescription to a given efficacy, and the corresponding attentive weights of the herbs in the prescription expressing their correlations to the efficacy. For a given efficacy, we collect prescriptions with the efficacy as positive samples and prescriptions without the efficacy as negative samples, and train our hierarchical attentive neural network with these samples. Afterwards, the trained model is used to distill the positive samples with the following strategy: the herbs with attentive weights below a prespecified threshold in a positive sample prescription are treated as being unrelated to the efficacy and thus filtered 


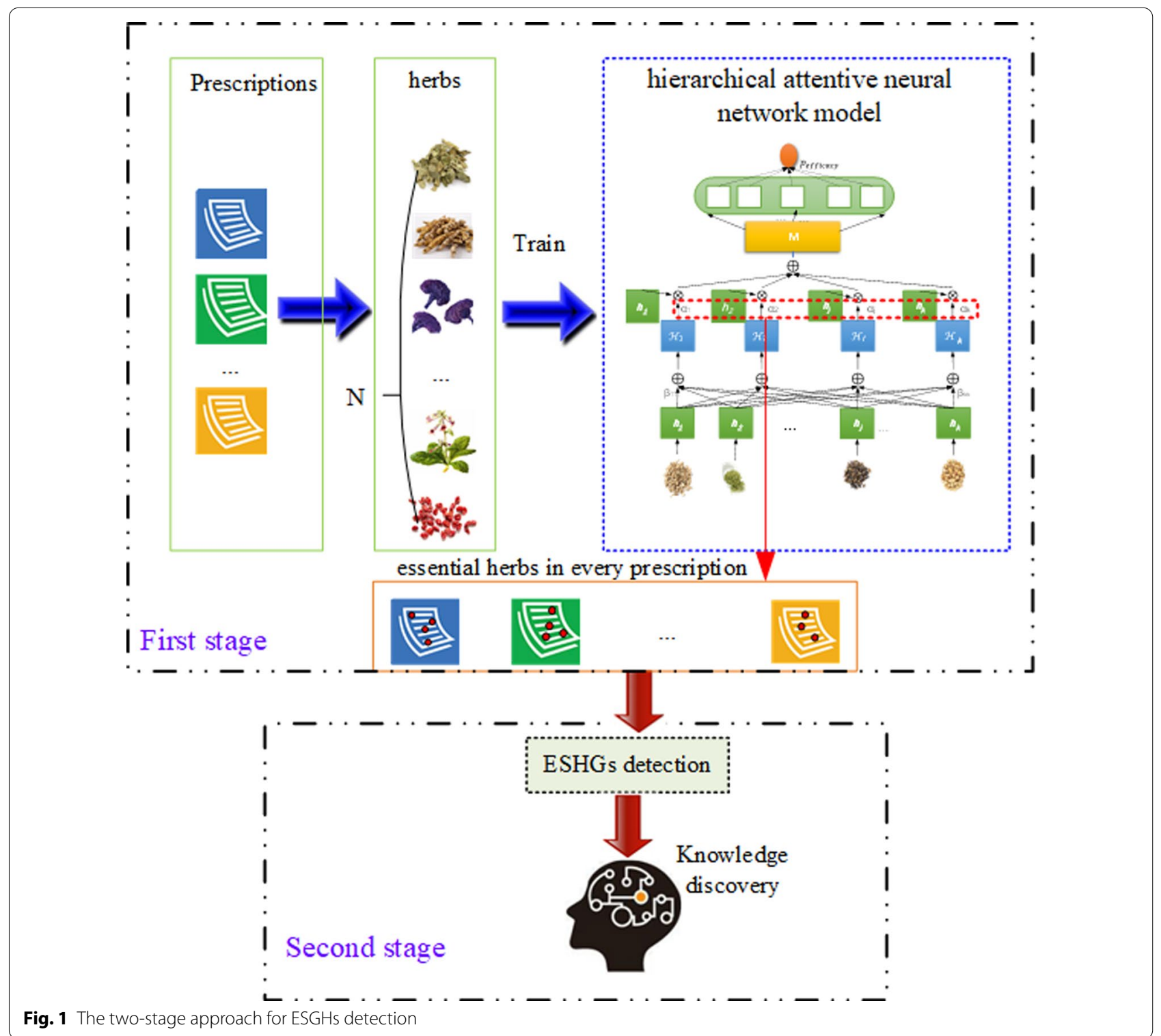

out from the prescription, and the remaining herbs are thought as essential in the prescription for its efficacy. The distilling process in the first stage is then followed by the second stage, as shown in Fig. 1, where association analysis is performed to mine frequent herbal sets in the distilled positive sample collection and the frequent sets are returned as ESHGs of the efficacy.

\section{Hierarchical attentive neural network model}

The hierarchical attentive neural network devised to identify essential herbs in a prescription for its efficacy is shown in Fig. 2. The model is divided into four layers, namely a herb embedding layer, two attention layers and an output layer. We detail these layers separately below.
Let $H \in \mathbb{R}^{N \times d^{h}}$ be the embedding vocabulary with $N$ rows corresponding to distinct herbs of TCM, which are expressed in $H$ with real-valued vectors of dimensionality $d^{h}$. For a given prescription $P=\left\{h_{1}, h_{2}, \ldots, h_{k}\right\}$ consisting of $k$ distinct herbs $h_{1}, h_{2}, \ldots$, and $h_{k}$,where $h_{i}$ is denoted with the corresponding row index in $H$, the herb embedding layer of our model first extract those row vectors indexed with $h_{1}, h_{2}, \ldots$, and $h_{k}$ from $H$ (i.e. the embeddings of those herbs in $H$ ), and stacks them into a matrix $\mathcal{H} \in \mathbb{R}^{k \times d^{h}}$ as the initial representation of $P$ for our model. Afterwards, the matrix $\mathcal{H}$ is fed to the two attention layers, where the first attention layer aims to capture correlations among herbs in $P$ and enrich every herb's embedding in $\mathcal{H}$ with the correlational 


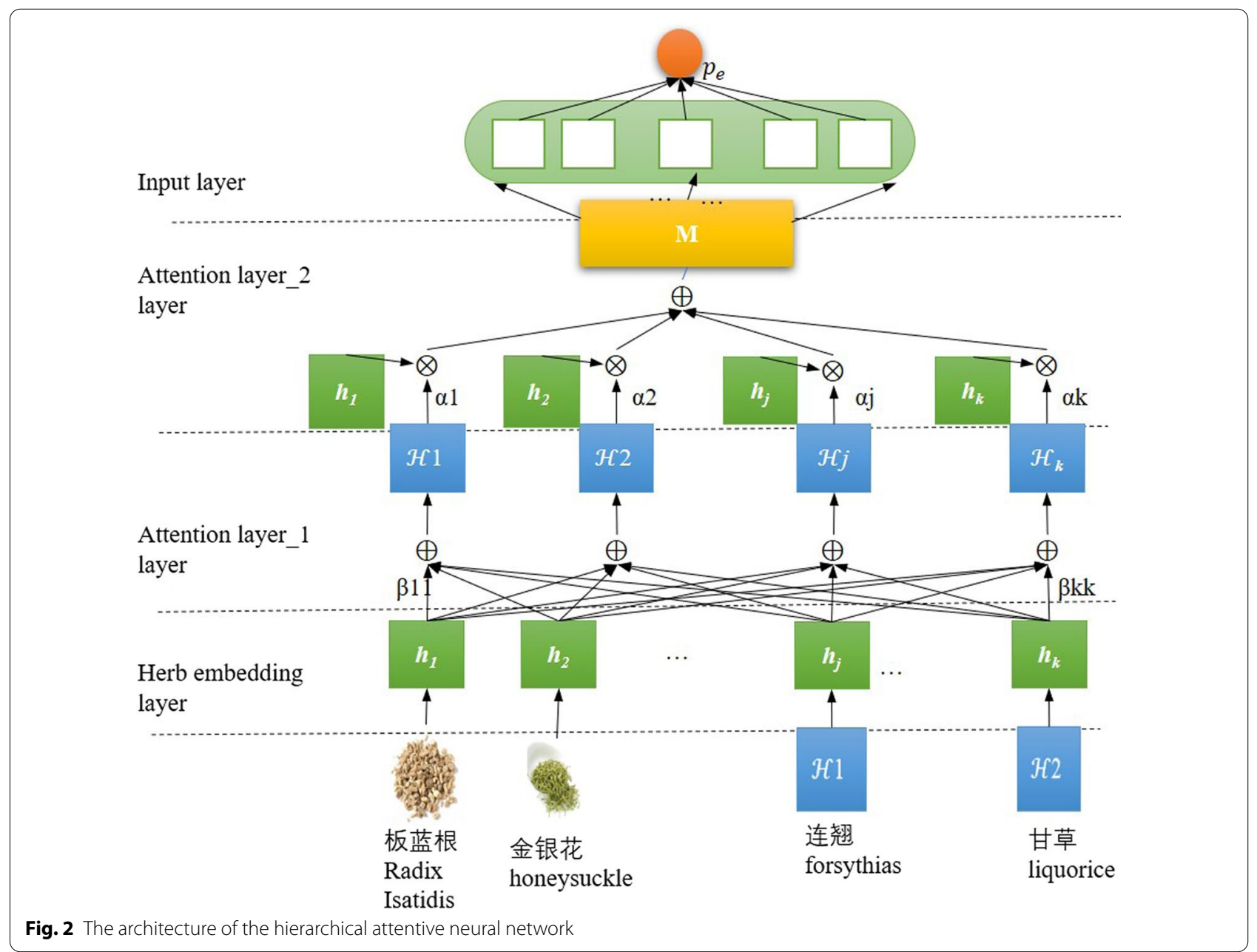

information, while the second attention layer attempts to distinguish essential herbs of $P$ for its efficacy which are verified by the last layer, i.e. the output layer. The two attention layers together transform the initial representation $\mathcal{H}$ of a prescription into a compact vector representation of the prescription to which all the herbs in the prescription contribute in accordance with their final attentive weights (i.e. obtained in the second attention layer), respectively. The last output layer takes the compact representation and outputs a probability of the prescription belonging to its corresponding efficacy. The training algorithm based on gradient descent adapts the parameters in our model to maximize the probability. The attentional weights in the second attention layer for a prescription resulted from the learned model parameters are employed to distill the prescription.

\section{The first attention layer}

The purpose of the first attention layer is to capture correlations among herbs in a prescription and accordingly update its initial representation in $\mathcal{H}$. Concretely, given a prescription $P$ and its initial representation $\mathcal{H}$, this layer first calculates attentive weights of a herb over all the herbs in $P$ to form a weight distribution vector of the dimensionality $k$, and all such vectors of the $k$ distinct herbs are collected into the matrix $\beta \in \mathbb{R}^{k \times k}$, as shown in formula (1), where $W_{p 1} \in \mathbb{R}^{d^{h} \times d^{h}}$ is the parameter of the model to be learned in the training process. After that, all the initial representation vectors in $\mathcal{H}$ are summarized in $P$ according to the weight distribution vector of an herb over all the herbs of $P$, resulting in an updated representation for the herb. Formula(2) gives the calculation for all the herbs in a prescription, where $\overline{\mathcal{H}} \in \mathbb{R}^{k \times d^{h}}$ contains the updated representations for all the herbs in $P$.

$$
\beta=\operatorname{softmax}\left(\mathcal{H} W_{p 1} \mathcal{H}^{T}\right)
$$

$$
\overline{\mathcal{H}}=\beta \mathcal{H}
$$




\section{The second attention layer}

The second layer takes the updated representations for all the herbs of $P$ in $\overline{\mathcal{H}}$, captures contributions of these herbs for the efficacy of $P$ and accordingly formulates feature vector of $P$ for fitting its efficacy. The contributions are characterized with the learned weight distribution $\alpha$ in formula (3), where $W_{p 2} \in \mathbb{R}^{d^{a} \times d^{h}}$ and $W_{p 3} \in \mathbb{R}^{1 \times d^{a}}$ are learnable parameters of the model, and $\alpha \in \mathbb{R}^{k}$ is the weights of all the herbs in $P$ for its efficacy. According to these weights, the feature vector $M \in \mathbb{R}^{d^{h}}$ of $P$ for fitting its efficacy is calculated with the weighted sum of all the herbal embeddings in $H$, as shown in formula (4).

$$
\begin{aligned}
& \alpha=\operatorname{softmax}\left(W_{p 3} \tanh \left(W_{p 2} \overline{\mathcal{H}}^{T}\right)\right. \\
& M=\alpha \mathcal{H}
\end{aligned}
$$

A prescription, which is treated as a collection of herbs, is mapped to a low-dimensional space by utilizing the weighted sum of all its herbal embeddings, and its feature vector has the same dimensionality as herbal embedding.

\section{The output layer and the loss function}

The final layer, i.e. the output layer, is a typical perceptron, which performs affine transformation on the $M$ and the resulted score is squashed into a probability of the prescription belonging to its efficacy. The process is described as in formula (5), where $W_{p 4} \in \mathbb{R}^{d^{m} \times d^{h}}$ and $W_{p 5} \in \mathbb{R}^{d^{h} \times 1}$ are learnable parameters of the model, and $\mathrm{p}_{e} \in[0,1]$ is the probability.

$$
p_{e}=\operatorname{sigmoid}\left(W_{p 5} \operatorname{ReLU}\left(W_{p 4} M^{T}\right)\right.
$$

The training objective for our model is the standard cross-entropy, shown in formula (6), and the stochastic gradient descent algorithm is employed to achieve the optimization. In formula (6), $y \in\{1,0\}$ is the groundtruth efficacy label of a prescription with 1 denoting the target efficacy for which we are mining ESHGs, and 0 denoting the other efficacies. In addition, we leverage $L_{2}$ norm of all the model parameters (expressed here with $\omega)$ to regularize the cross-entropy loss, and $\lambda$ is the corresponding hyperparameter.

$$
\begin{aligned}
L= & -\sum\left[y \log \left(p_{e}\right)+(1-y) \log \left(1-p_{e}\right)\right] \\
& +\lambda\|\omega\|_{2}
\end{aligned}
$$

Figure 3 depicts the whole flow of the hierarchical attentive neural network model and different parameters employed therein, including their dimensionalities and the alignments in the dimensionalities.

\section{ESHGs detection}

In order to detect ESHGs for an efficacy, we collect prescriptions with the efficacy as the positive samples and prescriptions without the efficacy as negative samples, and all of them compose our training data set for the efficacy. When convergence emerges in the training of our model, all the herbs in a positive sample with their corresponding attention weight $\alpha_{i}$ below a prespecified threshold are filtered out and the remaining herbs formulate the corresponding distilled prescription. All the distilled prescriptions for a given efficacy obtained with such manner are fed to the Apropri algorithm for mining frequent herbal sets and such frequent sets are regarded as ESHGs for the given efficacy.

\section{Results}

Data sets

Empirical evaluation of our method requires two types of data. The first one is the efficacies with their known corresponding ESHGs as the gold standard data, and the second one is the prescriptions accompanying with their efficacies as the data to be mined for detecting ESHGs. Due to the lack of gold standard data widely accepted for our task, we intentionally collect efficacies having known reliable ESHGs from Internet, and invite a TCM expert to evaluate these collected ESHGs, finally obtain a data set of 14 efficacies with their corresponding ESGHs for our evaluation. For instance, the efficacy "activating blood to resolve stasis(活血化瘀)" has a known ESHG “红花,桃仁，川芦(Flos Carthami, Semen Persicae, Rhizoma Ligustici Chuanxiong)". For the second type of data, we extract the prescriptions having the efficacies in the gold standard dataset from the prescription database ${ }^{1}$ and thus construct the dataset to be mind to detect ESGHs for the efficacies in the standard dataset.And the numbers and the examples of ESGHs of 14 efficacies are given in Table 1.

For a specific efficacy, we treat prescriptions with the efficacy as positive samples and prescriptions without the efficacy as negative samples. Because of the data skew for positive and negative samples, we utilize an under-sampling method to obtain a balanced dataset in a ratio of positive to negative samples of 1:1 for each efficacy. And the numbers of prescriptions extracted for each of the 14 efficacies are listed in Table 2.

\section{Evaluation method}

The purpose of our work is to capture the essential herbs in a prescription for a given efficacy by a classification process and then discover ESHGs from the essential 


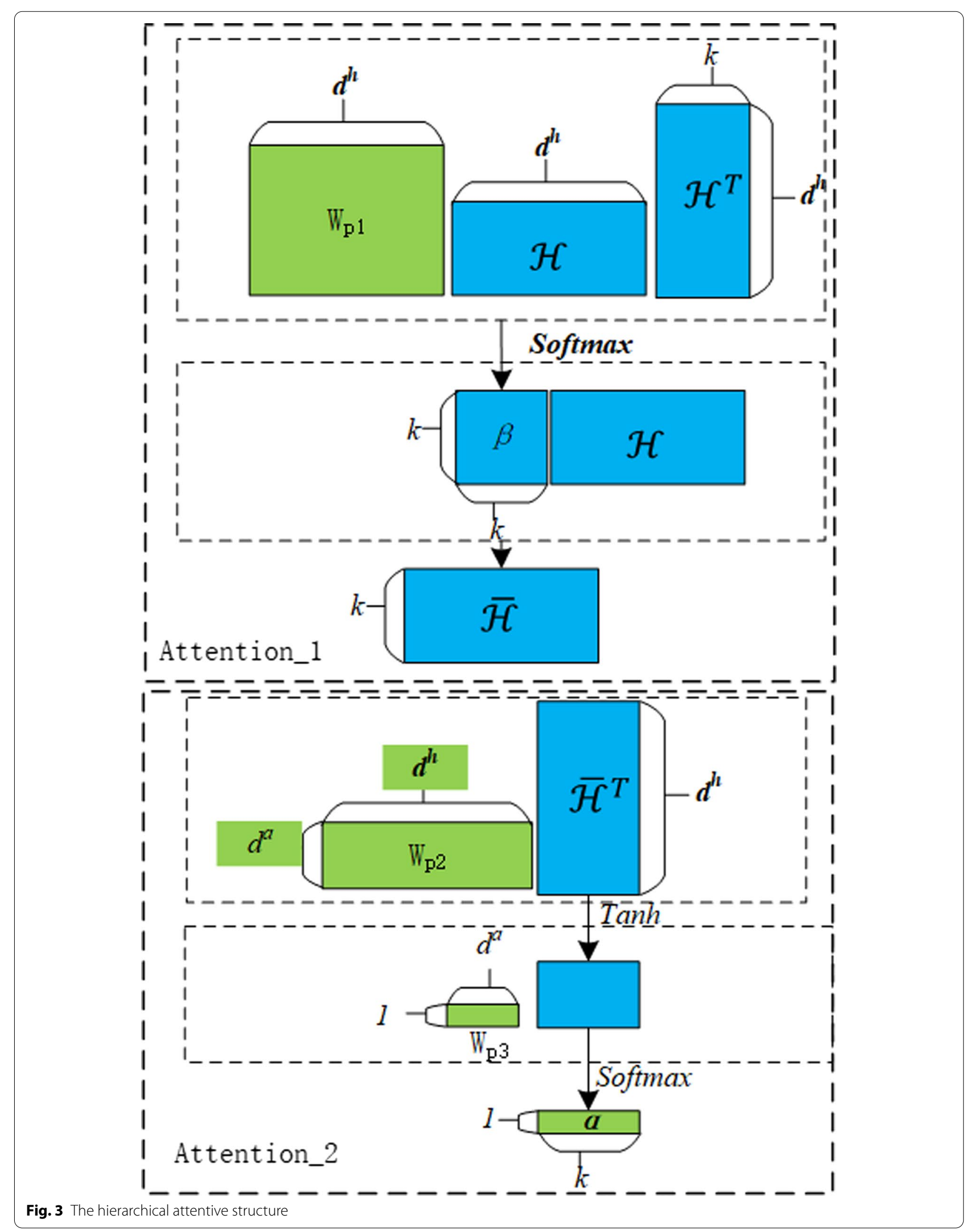


herbs in prescriptions. Hence, the evaluation metrics are the accuracies of the discovered ESHGs instead of the classification. Due to the lack of gold-standard data widely accepted for our task, we could not find out all possible ESHGs for calculating the recall metric.

For a given efficacy, there are multiple gold standard ESHGs, as shown in Table 1, and our algorithm is also likely to generate multiple ESHGs for it according to the support threshold. In order to evaluate the effectiveness of the generated ESHGs, we have to compare them against the corresponding gold standard ESHGs for the same efficacy. Obviously, the evaluation is a comparison between two sets, therefore, we propose to employ Dice coefficient for the evaluation. To be specific, suppose $e$ is an efficacy with its gold standard ESHGs $S^{e}, A^{e}$ is the ESHGs returned by our algorithm for $e$, and the Dice coefficient on $e$ is

$$
\text { Dice }_{e}=\frac{2\left|A^{e} \bigcap S^{e}\right|}{\left|A^{e}\right|+\left|S^{e}\right|}
$$

Because $\left|S^{e}\right|$ is fixed in the evaluation process, we ignore it and the formula (7) becomes

$$
A c c_{e}=\frac{\left|A^{e} \bigcap S^{e}\right|}{\left|A^{e}\right|}
$$

The identification accuracy on the whole gold standard test set is

$$
A c c_{e}=\frac{1}{14} \sum_{e=1}^{14} A c c_{e}
$$

The above metric is in essence exact-matching-based, i.e. a pair of ESHGs from two sets respectively have to be matched exactly in order to contribute to the accuracy. The exact matching requirement is unreasonable because it treats equally both of the partial matching and mismatching situations, thus it is necessary to incorporate different matching situations into unified accuracy measurement. But unfortunately, the solution is not so obvious due to that we have no any knowledge about alignment between the two sets of ESHGs to be compared. In this paper we employ a greedy strategy with which an ESHG from $\mathrm{A}^{e}$ is aligned with the ESGH from $S^{e}$ who has maximum overlapping with the ESHG from $\mathrm{A}^{e}$ in their contained herbs. Formally, given an ESHG $h_{g}$ from $\mathrm{A}^{e}$, its correctness relative to $S^{e}$ is defined as formula (10).

$$
\text { Correctness }(h g)=\max _{h g^{\prime} \in S^{e}} \frac{2\left|h g \bigcap h g^{\prime}\right|}{|h g|+\left|h g^{\prime}\right|}
$$

where $\left|h g \bigcap h g^{\prime}\right|$ is the number of the same herbs in $h g$ and $h g^{\prime} .|h g|$ and $\left|h g^{\prime}\right|$ are the herbal numbers in two ESHGs, respectively.

Based on formula (10), the identification accuracy of our algorithm for a given efficacy $e$, when it identifies $A^{e}$, but the corresponding gold standard ESHGs is $S^{e}$, is adapted from formula (8) to formula (11).

$$
A c c_{e}=\frac{\sum_{h g \in A^{e}} \text { Correctness }(h g)}{\left|A^{e}\right|}
$$

We finally utilize the formula (9) where the $A c c_{e}$ is calculated through formula (11) to evaluate the effectiveness of our algorithm.

\section{The hyperparameters}

In order to determine the optimal hyperparameters in our model, we leave the efficacy "heat-clearing and detoxifying(清热解毒)” with its gold standard ESHGs and the corresponding prescriptions out of the data as our development dataset, and based on the development, we set the hyperparameters of our model as shown Table 3. Moreover, the herb embedding vocabulary $H$ is initialized randomly, and the attentive weights in 10 runs of the training process are averaged as the final attentive weights of herbs in a prescription to discover essential herbs in the prescription for its efficacy.

\section{The effect of ESHGs detection}

For a given efficacy, its positive and negative samples first pass through the hierarchical attentive neural network and with the model all herbs in these samples obtain their corresponding attentive weights relative to the sample prescription they belong to. After that, all herbs in a positive prescription are ranked in descending order of their attentive weights, and the top $N$ herbs are chosen to form the corresponding distilled prescription. In order to improve the quality of the distilled prescriptions, the positive and negative samples are fed into the hierarchical attentive neural network 10 times with its different random initializations of the parameters, thus resulting in 10 groups of the attentive weights. Afterwards, all the 10 attentive weights for an herb in a positive prescription are summed, and the herbs in a positive prescription are ranked in descending order of their summed attentive weights, and the top $N$ herbs are retained as the elements of the corresponding distilled prescription.

The resultant distilled prescriptions are then fed to the Apriori algorithm for mining ESHGs of the efficacy. We investigate different settings for the parameter $N$, and also for $K$, the size of an itemset in the Apriori algorithm, and the support threshold min_sup in the 


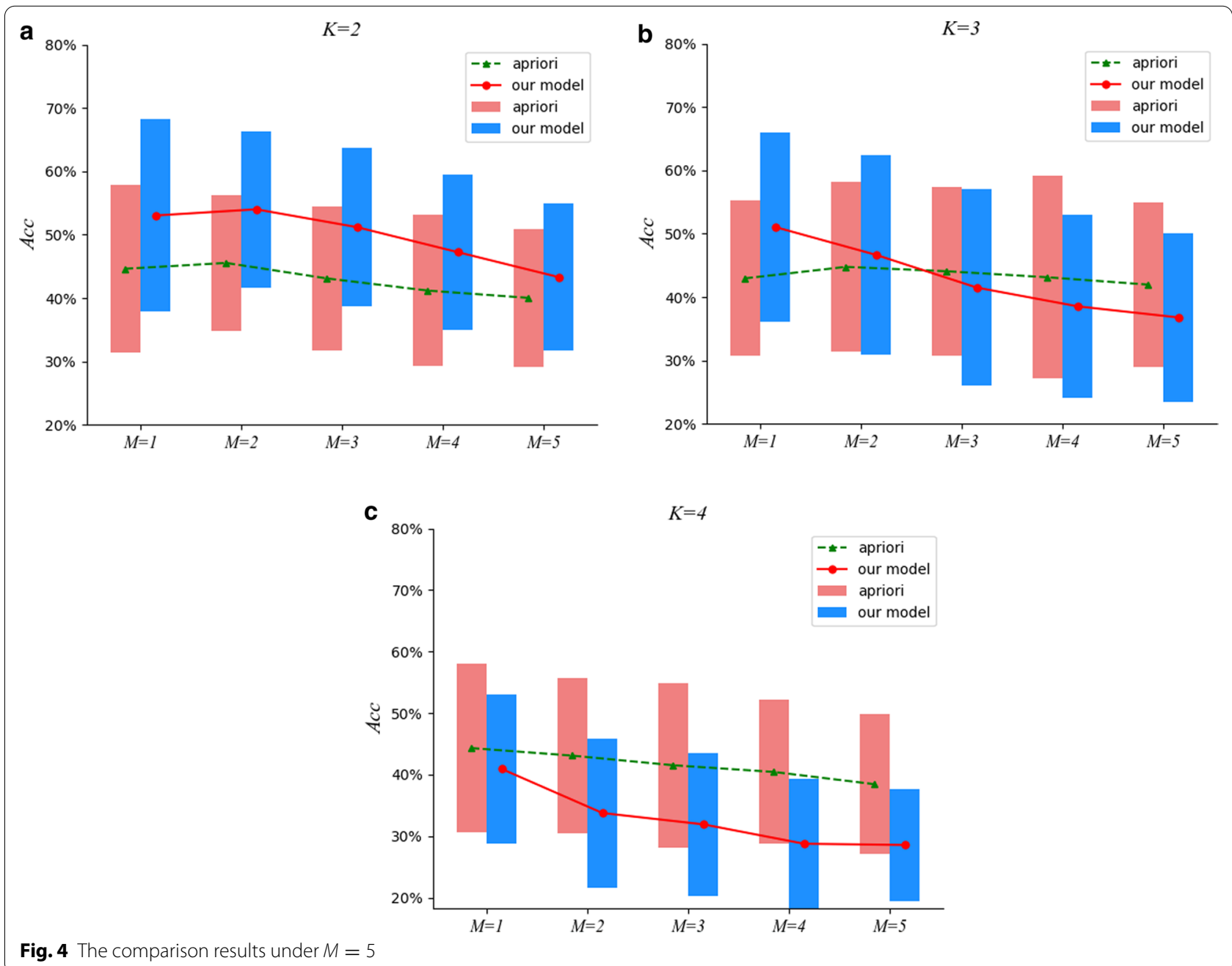

Apriori algorithm. Furthermore, we also run the Apriori algorithm with the same parameter settings (i.e. the $K$ and min_sup) on all the positive prescriptions without the distilling process, and compare the results to the corresponding results obtained with the distilling process. Furthermore, in order to verify the stability of our two-stage approach, we perform the two-stage processing 10 times on the same positive and negative samples, and the 10 experimental results in terms of $A c c_{e}$ for an efficacy are averaged. The average $A c c_{e}$ and the corresponding 95\% confidence interval are reported hereafter to represent the effectiveness of the approach for an efficacy.

Table 4 gives the average $A c c_{e}$ of our two-stage approach for the 13 test efficacies, the whole average $A c c_{e}$ for all these efficacies and the corresponding performances of the simple Apriori algorithm without the distilling process under the conditions $N=8$ and min_sup $=0$ combined with different $K \in\{2,3,4\}$.
The experimental results in Table 4 shows that our two-stage approach with the distilling process based on the hierarchical attentive neural network consistently outperforms the counterpart approach based on the simple Apriori without the distilling process. Our hierarchical attentive neural network employs two attentive layers to capture the correlations among herbs in a prescription as well as essential herbs in a prescription for its efficacy, which makes the distilled prescription cleaner and clearer in its efficacy description, thus improving the ESHGs detection based on the Apriori algorithm. For instance, for the efficacy "activating blood to resolve stasis (活血化瘀", without the distilling process the number of the frequent 2-itemsets (i.e. $K=2$ ) reaches to about 5230 , but the distilling process reduces the number to about 2400 and at the same time the accuracy in the ESHGs detection increases 5.48\%. A slight exception among the accuracies of Table 4 arises on the efficacy “relieving pain(止痛)" due to the small-sized positive 
Table 1 The numbers of ESHGs and examples for each efficacy

\begin{tabular}{|c|c|}
\hline Efficacy name & NumExamples of Gold-stand ESHGs \\
\hline $\begin{array}{l}\text { 活血化瘀 } \\
\text { activating blood } \\
\text { to resolve stasis }\end{array}$ & $\begin{array}{l}10 \text { 红花,桃仁,牛膝(Flos Carthami, Semen Persicae, Radix Achyranthis Bidentatae) } \\
\text { 水蛭,三棱,我(Hirudo, Rhizoma Sparganii, Rhizoma Curcumae) } \\
\text { 丹皮,赤莐,桃仁(Cortex Moutan Radicis, Radix Paeoniae Rubra,Semen Persicae) } \\
\text { 红花,桃仁,川芎(Flos Carthami, Semen Persicae, Rhizoma Ligustici Chuanxiong) }\end{array}$ \\
\hline 化痰dispersing phlegm & $\begin{array}{l}19 \text { 陈皮,获苓,半夏(Pericarpium Citri Reticulatae, Poria, Rhizoma Pinelliae) } \\
\text { 紫菀,桑白皮,亭苭(Radix Asteris, Cortex Mori, Semen Lepidii)) } \\
\text { 贝母,桔梗,甘草(Bulbus Fritillaria, Radix Platycodonis, Radix Glycyrrhizae } \\
\text { 款冬花,枇杷叶,紫菀(Flos Farfarae, Folium Eriobotryae, Radix Asteris) }\end{array}$ \\
\hline $\begin{array}{l}\text { 明目 } \\
\text { improving eyesight }\end{array}$ & 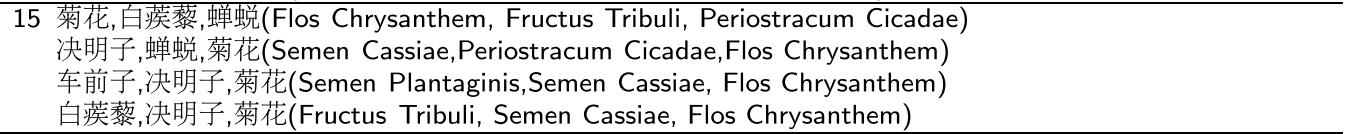 \\
\hline $\begin{array}{l}\text { 壮精骨 } \\
\text { strengthening bone }\end{array}$ & $\begin{array}{l}13 \text { 牛樌,骨碎补,狗脊(Radix Achyranthis Bidentatae, Rhizoma Drynariae, Rhizoma Cibotii) } \\
\text { 破故纸,续断,狗脊(Fructus Psoraleae, Radix Dipsaci,Rhizoma Cibotii) } \\
\text { 杜仲,寄生,续断(Cortex Eucommiae,Herba Visci Radix Dipsaci) } \\
\text { 杜仲,破故纸,狗脊(Fructus Psoraleae,Rhizoma Cibotii) }\end{array}$ \\
\hline $\begin{array}{l}\text { 止血 } \\
\text { stopping bleeding }\end{array}$ & $\begin{array}{l}38 \text { 大黄,三七,白芨(Radix et Rhizoma Rhei, Radix Notoginseng, Bletilla striata) } \\
\text { 丹皮,侧柏叶,白茅根(Cortex Moutan Radicis, Cacumen Platycladi, Rhizoma Imperatae) } \\
\text { 仙鹤草,茜草,三七(Herba Agrimoniae, Radix Rubiae,Radix Notoginseng) } \\
\text { 侧柏叶,仙鹤草,血余炭(Cacumen Platycladi, Herba Agrimoniae,Crinis Carbonisatu) }\end{array}$ \\
\hline $\begin{array}{l}\text { 消肿 } \\
\text { diminishing swelling }\end{array}$ & $\begin{array}{l}18 \text { 桂枝,猪苓,泽泻(Ramulus Cinnamomi, Polyporus Umbellatus, Rhizoma Alismatis) } \\
\text { 玄参,牡蚛, 贝母(Radix Scrophulariae, Concha Ostreae, Bulbus Fritillaria) } \\
\text { 瓜蒌,贝身,蒲公英(Fructus Trichosanthis, Bulbus Fritillaria,Herba Taraxaci) } \\
\text { 桑白皮,生姜,茯苓(Cortex Mori, Rhizoma Zingiberis Recens, Poria) } \\
\end{array}$ \\
\hline $\begin{array}{l}\text { 利湿 } \\
\text { draining dampness }\end{array}$ & $\begin{array}{l}29 \text { 䄷子,黄柏,茵陈(Fructus Gardeniae, Cortex Phellodendri, Herba Artemisiae Scopariae) } \\
\text { 茵陈,伌子,大黄(Herba Artemisiae Scopariae,Fructus Gardeniae, Radix et Rhizoma Rhei) } \\
\text { 茵陈,金钱草,虎杖(Herba Artemisiae Scopariae,Herba Lysimachiae, Rhizoma Polygoni Cuspidati) } \\
\text { 薏仁,滑石,通草(coix seed, Talcum, Medulla Tetrapanacis) } \\
\end{array}$ \\
\hline $\begin{array}{l}\text { 健脾 } \\
\text { invigorating spleen }\end{array}$ & $\begin{array}{l}28 \text { 黄芪,白术,党参(Radix Astragali seu Hedysari, Rhizoma Atractylodis Macrocephalae, Radix Codonopsis) } \\
\text { 黄芪,白术,获苓(Radix Astragali seu Hedysari, Rhizoma Atractylodis Macrocephalae, Poria) } \\
\text { 白术,干姜,甘草(Rhizoma Atractylodis Macrocephalae, Rhizoma Zingiberis Radix Glycyrrhizae) } \\
\text { 山药,苂实,白术(Rhizoma Dioscoreae,Semen Euryales,Rhizoma Atractylodis Macrocephalae) }\end{array}$ \\
\hline $\begin{array}{l}\text { 止咳 } \\
\text { arresting cough }\end{array}$ & $\begin{array}{l}17 \text { 紫苏子,枇杷叶,杏仁(Fructus Perillae,Folium Eriobotryae,Semen Armeniacae Amarum) } \\
\text { 地龙,麻黄,杏仁(Lumbricus, Herba Ephedrae,Semen Armeniacae Amarum) } \\
\text { 杏仁,贝母,桑叶(Semen Armeniacae Amarum,Bulbus Fritillaria, Folium Mori) } \\
\text { 杏仁,枇杷叶,桑白皮(Folium Eriobotryae,Semen Armeniacae Amarum, Cortex Mori) } \\
\end{array}$ \\
\hline $\begin{array}{l}\text { 安神 } \\
\text { tranquillization }\end{array}$ & $\begin{array}{l}21 \text { 龙骨,零仁,远志(Os Draconis, Semen Ziziphi Spinosae, Radix Polygalae) } \\
\text { 远志,葛蒲,丹参(Radix Polygalae,Rhizoma Acori Tatarinowii, Radix Salviae Miltiorrhizae) } \\
\text { 小麦,获神,夜交藤(Fructus Tritici Levis,poria with hostwood, tuber fleeceflower stem) } \\
\text { 何首乌,夜交藤,零仁(Radix Polygoni Multiflori,tuber fleeceflower stem,Semen Ziziphi Spinosae) }\end{array}$ \\
\hline $\begin{array}{l}\text { 止痛 } \\
\text { relieving pain }\end{array}$ & $\begin{array}{l}11 \text { 蒲黄,五灵脂,玄胡(Pollen Typhae, Faeces Togopteri, Rhizoma Corydalis) } \\
\text { 五灵脂,玄胡,川棟(Faeces Togopteri, Rhizoma Corydalis, Fructus Meliae Toosendan) } \\
\text { 丹参,乳香,没药,当归(Radix Salviae Miltiorrhizae, Olibanum,Radix Angelicae Sinensis, Rhizoma Corydalis) } \\
\text { 玄胡,没药,五灵脂(Faeces Togopteri, Rhizoma Corydalis, Myrrha) }\end{array}$ \\
\hline $\begin{array}{l}\text { 止痒 } \\
\text { relieving itching }\end{array}$ & 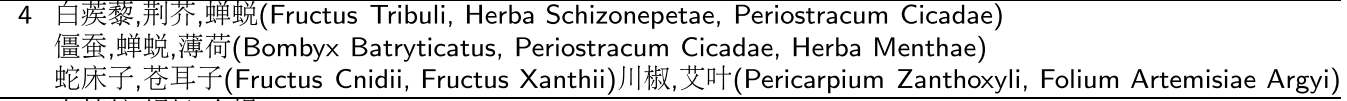 \\
\hline $\begin{array}{l}\text { 通络 } \\
\text { removing obstruction } \\
\text { in the channels }\end{array}$ & $\begin{array}{l}14 \text { 乌梢蛇,蜈蚣,全蝎(Zaocys, Scolopendra, Scorpio) } \\
\text { 秦艽,荅草,鸡血藤 (Radix Gentianae Macrophyllae, Herba Siegesbeckiae,Caulis Spatholobi) } \\
\text { 地龙,制川鸟, 制草乌,胆南星(Lumbricus, Rhizoma Arisaematis Cum Bile,Radix Aconiti } \\
\text { Kusnezofii Preparata, Radix Aconiti Preparata) } \\
\text { 地龙,当归,川芎(Lumbricus, Radix Angelicae Sinensis, Rhizoma Ligustici Chuanxiong) }\end{array}$ \\
\hline $\begin{array}{l}\text { 清热解毒 } \\
\text { heat-clearing and } \\
\text { detoxifying }\end{array}$ & 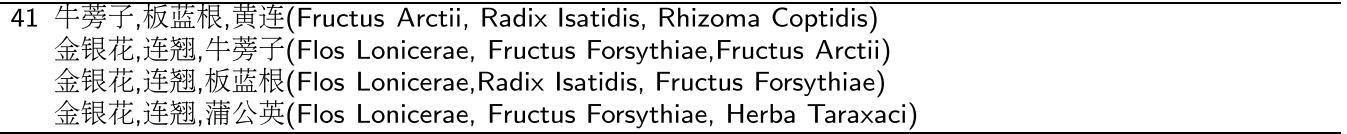 \\
\hline
\end{tabular}

Displayed is the number of instances, for each MTU group and all combined MTU, where the MTU quality evaluation metric was either (+) improved, $(-)$ worse, or $( \pm)$ no change. For each MTU group, sample size was 48 ( 8 MTU $\times 6$ subjects). For all combined MTU, sample size was 144 ( 8 per group $\times 3$ groups $\times 6$ subjects). Statistically significant differences are denoted using coloured cells, where green indicates improvement, red indicates worsening, and yellow indicates no change. In cases of no statistically significant difference between any of the three types of results, cells were left unshaded

+ , Represents improvement from models $1-3 ;-$, represents worsening from models $1-3 ; \pm$ represents no change between models 1 and 3 
Table 2 The numbers of prescriptions for each efficacy

\begin{tabular}{lc}
\hline Efficacy name & Num \\
\hline 活血化瘀activating blood to resolve stasis & 157 \\
化痰dispersing phlegm & 665 \\
明目improving eyesight & 277 \\
壮精骨strengthening bone & 168 \\
止血stopping bleeding & 225 \\
消肿diminishing swelling & 409 \\
利湿draining dampness & 206 \\
健脾invigorating spleen & 542 \\
止咳arresting cough & 182 \\
安神tranquillization & 246 \\
止痛relieving pain & 71 \\
止痒relieving itching & 151 \\
通络removing obstruction in the channels & 154 \\
清热解毒heat-clearing and detoxifying & 355 \\
\hline
\end{tabular}

Displayed is the number of instances, for each MTU group and all combined MTU, where the MTU quality evaluation metric was either (+) improved, (-) worse, or $( \pm)$ no change. For each MTU group, sample size was 48 ( 8 MTU $\times 6$ subjects). For all combined MTU, sample size was 144 ( 8 per group $\times 3$ groups $\times 6$ subjects). Statistically significant differences are denoted using coloured cells, where green indicates improvement, red indicates worsening, and yellow indicates no change. In cases of no statistically significant difference between any of the three types of results, cells were left unshaded

+ , Represents improvement from models $1-3 ;-$, represents worsening from models $1-3 ; \pm$ represents no change between models 1 and 3 dataset for this efficacy, which consists of just 71 samples. On the contrary, the efficacies "dispersing phlegm(化痰)" and "invigorating spleen(健脾)", have much more positive samples, therefore, the resultant ESHGs for them are also much more accurate.

Furthermore, we also investigate empirically the impact of different min_sup settings on the effectiveness of ESHGs detection. To be specific, we set min_sup to be the $M$-th largest support value among all the $K$-itemsets occurring in the prescription set, and inspect the effectiveness of the Apriori algorithm on the prescription set. Figures 4, 5, 6 and 7 demonstrate comparisons of our two-stage approach with the raw Apriori in the average effects of the 13 efficacies for various combinations of the parameter settings $M, K$ and $N$.

We can observe from Figs. 4, 5, 6 and 7 that, for $K=2$, our approach performs much better that the raw Apriori algorithm even we adopt aggressive $N$ (when $N$ is set to be a small value such as 5 , we aggressively filter the herbs in a prescription and retain at most only 5 herbs in a prescription). However, when $K=3$ and 4 , the aggressive

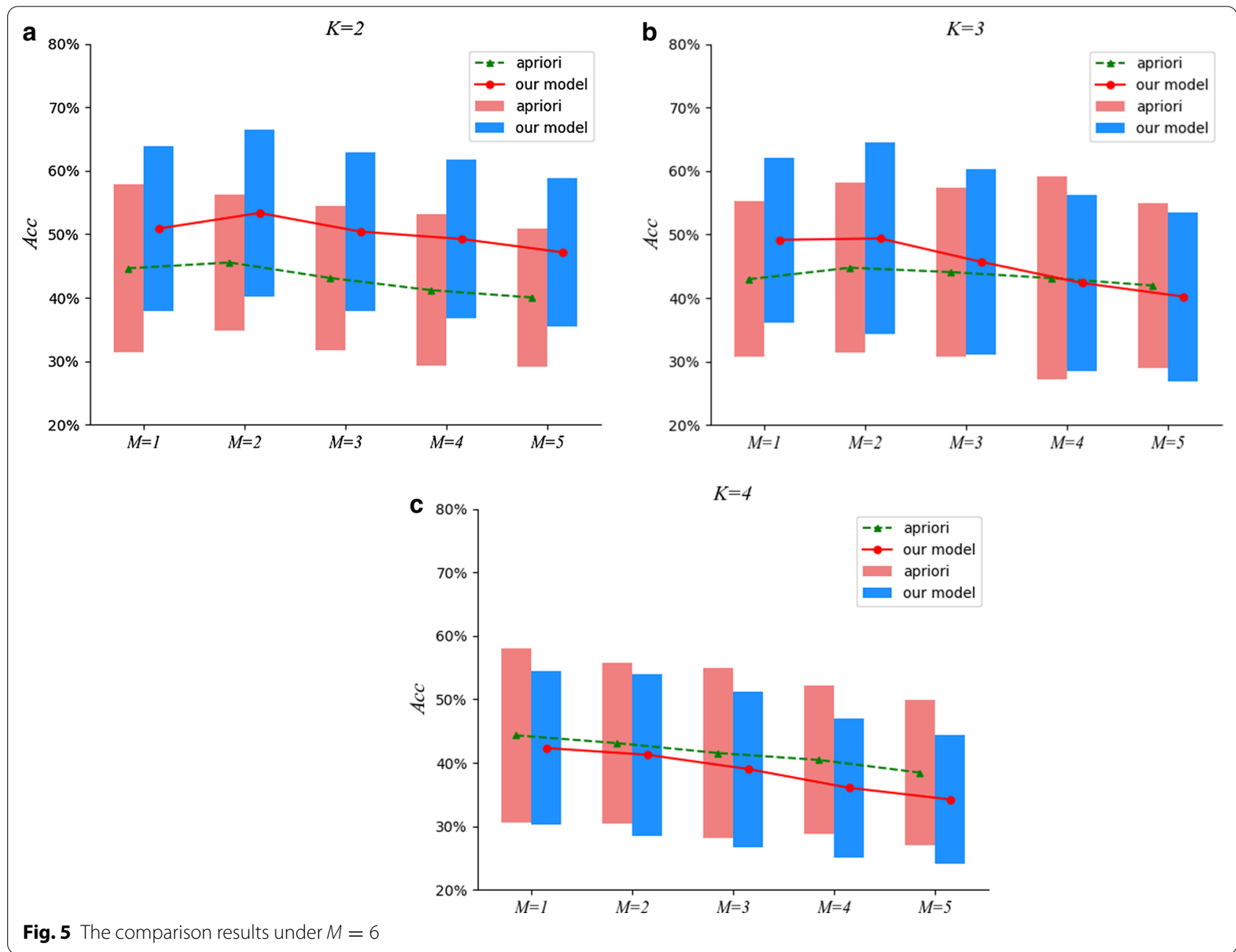



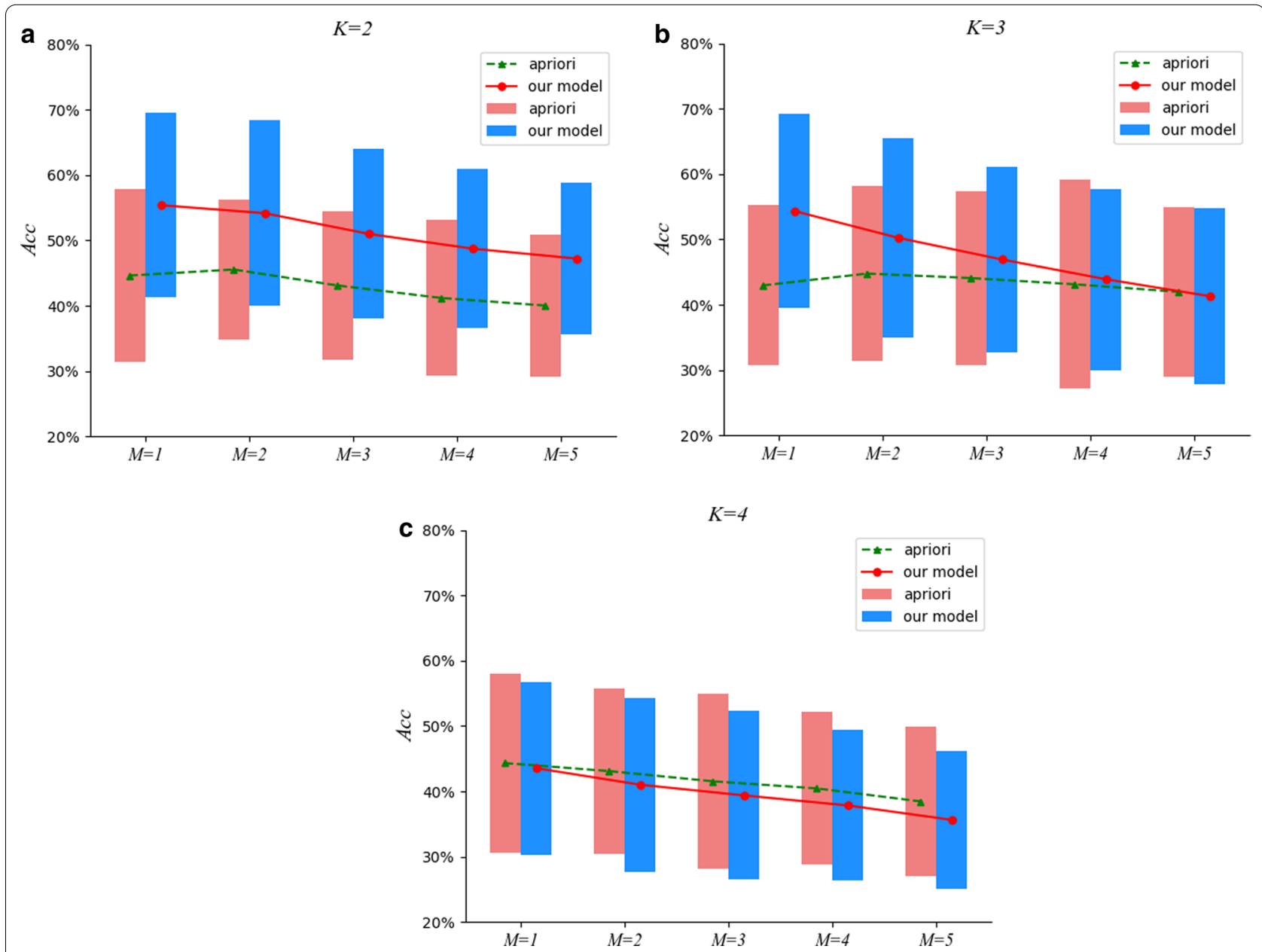

Fig. 6 The comparison results under $M=7$

distilling process leads to an obvious decrease in the performance. As we increase $N$ (for instance, $N=8$ ), the effectiveness is improved even when $K=3$ and 4 . Therefore, we reported the results in Table 4 with $N=8$.

\section{Effect of identifying essential herbs}

In this subsection, we further verify the effect of the hierarchical attentive neural network for capturing essential herbs in a prescription for its efficacy. For the purpose we collect some additional prescriptions with efficacies from the 14 ones in Table 1 . As the collecting results, we obtain 8 prescriptions for every efficacy from "activating blood to resolve stasis(活血化瘀”, “invigorating spleen(健脾)”, “arresting cough(止咳)” and “tranquillization(安神)", in total 32 prescriptions. We invite a TCM professional to annotate manually the essential herbs of these prescriptions for their corresponding efficacies. After that, we feed each of them into the trained hierarchical attentive neural network for the corresponding efficacy and therefrom fetch the attentive weights (i.e. the attentive weights of the second attentive layers) for the herbs in a prescription. We sort all the herbs in a prescription in a descending order of their attentive weights, compare them against the annotated essential herbs and accordingly evaluate the effect of our approach for the essential herb detection.

As for the evaluation metric, we employ a traditional one, namely MAP (Mean Average Precision), which is widely used for quantitative analysis of ranking algorithms in information retrieval and search engines. Suppose a prescription $p$ has $n_{p}$ essential herbs annotated by the TCM professional, the average precision $(A P)$ of the hierarchical attentive neural network on this prescription is defined as:

$$
A P_{p}=\frac{1}{n} \times \sum_{i=1}^{n_{p}} \frac{i}{\operatorname{position}(i)}
$$



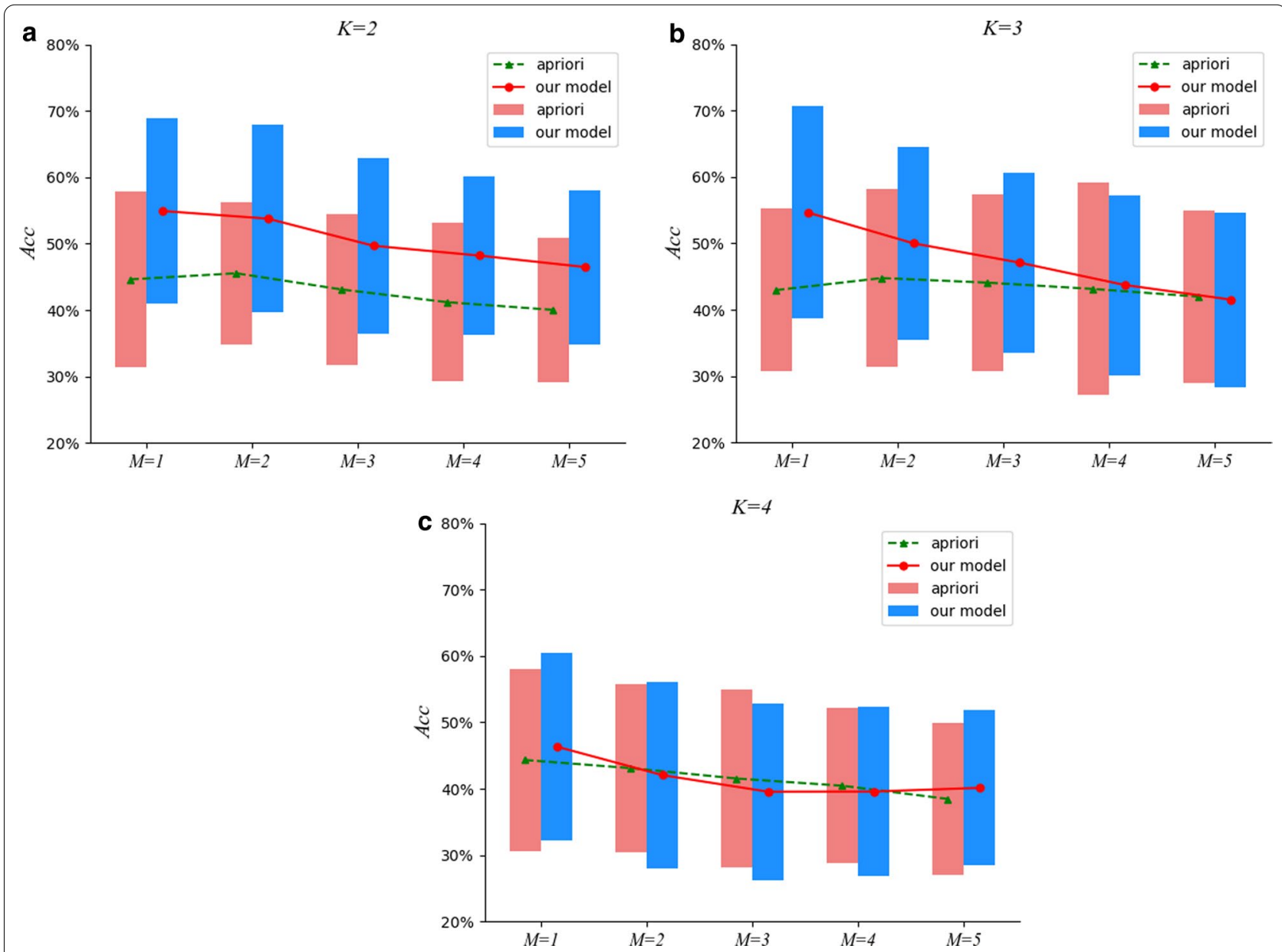

Fig. 7 The comparison results under $M=8$

Prescription:Radix Angelicae Sinens(当归), Radix Paeoniae Alba(白芶), Rhizoma Ligustici Chuanxiong(川芎), Radix Rehmanniae Preparata(熟地), Colla Corii Asini(阿胶), Radix Ginseng(人参), Cortex Albiziae(合欢皮), Semen Ziziphi Spinosae(酸柊仁), Poria(获苓), Radix Bupleuri(柴胡), Concha Ostreae(牡蚛) Efficacy: tranquillization(安神) Annotated essential herbs:Cortex Albiziae(合欢皮), Semen Ziziphi Spinosae(酸臬 仁), Poria(获苓), Concha Ostreae(牡蚛)

The attentive weights:

Radix Angelicae Sinens(当归), Radix Paeoniae Alba(白苟) Rhizoma Ligustici Chuanxiong(川芎)

Radix Rehmanniae Preparata(熟地) Colla Corii Asini(阿胶) Radix Ginseng(人参) Cortex Albiziae(合

欢皮) Semen Ziziphi Spinosae(酸疋仁) Poria(茯苓) Radix Bupleuri(柴胡), Concha Ostreae(牡蚛)

Fig. 8 A visualized demonstration of the attentive weights for a sample prescription

where position( $i$ ) is the position of the essential herb $i$ in the ranking list returned by our hierarchical attentive neural network for the prescription $p$. Furthermore, if an efficacy $e$ has $m_{e}$ prescriptions (here $m_{e}=8$ for all the four efficacies), the MAP of the neural network for $e$ is 
Table 3 The hyperparameter values

\begin{tabular}{ll}
\hline Hyper-parameter name & Value \\
\hline Epochs & 30 \\
batch_size & 1 \\
Herb embedding dimension $\mathrm{d}^{h}$ & 50 \\
Optimization algorithm & Adam \\
$\mathrm{d}^{a}$ & 128 \\
Hidden units of full connection layer & 50 \\
L2 regular factor & 0.0002 \\
Optimization learning rate & 0.001 \\
\hline
\end{tabular}

$$
M A P_{e}=\sum_{p=1}^{m_{e}} A P_{p}
$$

Table 5 demonstrates the effects of the hierarchical attentive neural network for detecting essential herbs in prescriptions in terms of $A P_{p}$ and $M A P_{e}$. In addition, in Fig. 8 we give a visualized demonstration of the attentive weights for a sample prescription with its herbs shaded differently to express their respective attentive weight values. Observing Table 5 and Fig. 8, we can conclude that our hierarchical attentive neural network indeed is able to capture essential herbs in a prescription for its efficacy, gaining more than $60 \%$ of the $A P_{p}$ for majority

Table 4 The effectiveness of our approach in comparison with the simple Apriori under $N=8$ and $\min \_$sup $=0$

\begin{tabular}{|c|c|c|c|c|c|c|}
\hline \multirow[t]{2}{*}{ Efficacy } & \multicolumn{3}{|c|}{ The simple Apriori } & \multicolumn{3}{|c|}{ Apriori on distilled prescriptions } \\
\hline & $K=2(\%)$ & $k=3(\%)$ & $k=4(\%)$ & $k=2(\%)$ & $k=3(\%)$ & $k=4(\%)$ \\
\hline Activating blood to & 10.30 & 14.09 & 14.32 & $15.78 \pm 0.18$ & $22.79 \pm 0.24$ & $25.77 \pm 0.25$ \\
\hline \multicolumn{7}{|l|}{ Resolve stasis } \\
\hline Dispersing phlegm & 15.45 & 21.34 & 23.17 & $18.08 \pm 0.16$ & $25.27 \pm 0.18$ & $28.53 \pm 0.22$ \\
\hline Strengthening bone & 8.51 & 11.83 & 13.36 & $11.56 \pm 0.14$ & $17.46 \pm 0.24$ & $20.42 \pm 0.34$ \\
\hline Stopping bleeding & 18.62 & 23.58 & 24.56 & $19.13 \pm 0.27$ & $23.75 \pm 0.42$ & $25.27 \pm 0.49$ \\
\hline Diminishing swelling & 5.99 & 6.77 & 6.68 & $6.58 \pm 0.15$ & $8.44 \pm 0.19$ & $9.42 \pm 0.20$ \\
\hline Draining dampness & 17.98 & 22.25 & 22.63 & $19.48 \pm 0.17$ & $23.99 \pm 0.20$ & $24.90 \pm 0.19$ \\
\hline Improving eyesight & 10.37 & 13.67 & 15.00 & $13.86 \pm 0.18$ & $19.37 \pm 0.20$ & $21.52 \pm 0.21$ \\
\hline Invigorating spleen & 17.12 & 24.46 & 27.09 & $21.32 \pm 0.17$ & $31.35 \pm 0.15$ & $36.21 \pm 0.21$ \\
\hline Arresting cough & 15.81 & 20.85 & 22.01 & $19.78 \pm 0.16$ & $27.52 \pm 0.26$ & $31.09 \pm 0.35$ \\
\hline Tranquillization & 15.78 & 20.76 & 21.97 & $19.90 \pm 0.11$ & $27.72 \pm 0.11$ & $31.90 \pm 0.13$ \\
\hline Relieving pain & 8.88 & 12.27 & 14.30 & $9.88 \pm 0.20$ & $12.92 \pm 0.28$ & $14.30 \pm 0.31$ \\
\hline Relieving itching & 7.74 & 10.81 & 11.77 & $8.39 \pm 0.22$ & $11.82 \pm 0.38$ & $13.47 \pm 0.46$ \\
\hline Removing obstruction & 14.62 & 19.68 & 20.91 & $18.72 \pm 0.07$ & $25.00 \pm 0.09$ & $26.93 \pm 0.08$ \\
\hline \multicolumn{7}{|l|}{ in the channels } \\
\hline The whole average & 12.86 & 17.11 & 18.29 & 15.57 & 21.34 & 23.83 \\
\hline
\end{tabular}

Table 5 The average precision of each essential-herb-labeled prescription

\begin{tabular}{lllll}
\hline Prescription & \multicolumn{1}{l}{$\begin{array}{l}\text { Activating blood to resolve stasis } \\
(\%)\end{array}$} & Invigorating spleen (\%) & Arresting cough cough (\%) & $\begin{array}{l}\text { Tranquillization } \\
\text { (\%) }\end{array}$ \\
\hline 1 & 100.00 & 100.00 & 57.42 & 100.00 \\
2 & 91.51 & 95.00 & 38.54 & 100.00 \\
3 & 55.97 & 74.09 & 72.09 & 58.33 \\
4 & 60.95 & 75.76 & 51.44 & 100.00 \\
5 & 77.08 & 94.29 & 65.12 & 48.89 \\
6 & 72.92 & 100.00 & 57.78 & 9.55 \\
7 & 63.54 & 83.33 & 81.00 & 59.03 \\
$M A P_{e}$ & 76.95 & 88.57 & 64.24 & 66.86 \\
\end{tabular}


of the prescriptions. The extreme exception arises for the sixth prescription of the efficacy “tranquillization(安神)", for which the $A P_{p}$ is only $9.55 \%$. In order to get insight into the reason of the extreme exception, we analyze manually the prescription and find that, it is composed of herbs "Rhizoma Ligustici Chuanxiong(川芦), Radix Paeoniae Alba(白苻), Radix Rehmanniae Recens(生地 ), Caulis Spatholobi(鸡血藤), Folium Mori(桑叶), Flos Chrysanthem(菊花), Tribulus terrestris(刺 静藜), Herba Mentha(薄荷), Rhizoma et Radix Notopterygi(㒸活), Semen Ziziphi Spinosae(酸零仁), Radix Ginseng (人参)" and labeled wrongly with the auxiliary efficacy "tranquillization(安神)", but in fact its primary efficacy should be “clearing liver heat and restraining liver yang(清肝平肝)". The label noise results in the erroneous essential herbs identified by the hierarchical attentive neural network.

\section{Discussion}

In summary, we utilize a neural network with two attentive layers to identify essential herbs in a prescription for its efficacy, and then discover ESHGs from TCM prescriptions. The efficacy of a prescription is very complicated which is related to herbal combination, herbal dosage, and dosage form. There are still some factors we have not considered. As a successor of this work, we intend to incorporate more herbal information such as dosage into our model to further lift the performance. Furthermore, discovering regularities in TCM prescription composition with data-driven methods and leveraging such regularities to guide the ESHGs detection are another our effort direction. Additionally, integrating the principle of Jun-Chen-Zuo-Shi into our model is another interesting point in our further study.

\section{Conclusion}

In this paper we propose a two-stage approach for discovering ESHGs from TCM prescriptions. We devise a neural network with two attentive layers to capture semantical correlations among herbs in a prescription and at the same time identify essential herbs in a prescription for its efficacy. Such attentions are beneficial to overcoming the difficulties when performing data mining on the prescription data accumulated in the long period of TCM history. The detailed experiments verify the effectiveness of our two-stage approach in the whole as well as the hierarchical attentive neural network for identifying the essential herbs in a prescription for its efficacy.

\section{Abbreviations}

ESHG: Efficacy-specific herbal group; TCM: Traditional Chinese medicine; LDA: Latent dirichlet allocation; MAP: Mean average precision.

Acknowledgements

Not applicable.

\section{Authors' contributions}

Conceptualization and Methodology, CL and LL; Review and Editing, CL and LL; Data collection and analyzation,ZY, YH, and LM; Experiment,ZY,YP,LL and LM; all authors read and approved the final manuscript.

\section{Funding information}

No funding was obtained for this study.

\section{Availability of data and materials}

The datasets used and analyzed during the current study are available from the first author upon reasonable requests.

\section{Ethics approval and consent to participate}

Not applicable.

Consent for publication

Not applicable.

\section{Competing interests}

The authors declare that they have no competing interests.

\section{Author details}

${ }^{1}$ Department of Computer Science, Sichuan University, Chengdu, China.

${ }^{2}$ School of Basic Medicine, Chengdu University of TCM, Chengdu, China.

Received: 21 July 2020 Accepted: 28 January 2021

Published online: 18 February 2021

\section{References}

1. Yao L, Zhang Y, Wei B, Wang W, Zhang Y, Ren X, Bian Y. Discovering treatment pattern in traditional chinese medicine clinical cases by exploiting supervised topic model and domain knowledge. Journal of Biomedical Informatics. 2015;58:260-7.

2. Li G, Liu B. Big data is essential for further development of integrative medicine. Chinese Journal of Integrative Medicine. 2015;21:323-31.

3. Liu B, Zhou X, Wang Y, Hu J, He L, Zhang R, Chen S, Guo Y. Data processing and analysis in real-world traditional chinese medicine clinical data: challenges and approaches. Statistics in Medicine. 2012;31:653-60.

4. Leem J, Jung W, Kim Y, Kim B, Kim K. Exploring the combination and modular characteristics of herbs for alopecia treatment in traditional chinese medicine: an association rule mining and network analysis study. Bmc Complementary \& Alternative Medicine. 2018;18:204-16.

5. Dai Z, Han Y. Study on application of data mining technology in syndrome differentiation of tcm. In: 2013 2nd International Conference on Systems Engineering and Modeling (ICSEM-13), beijing; 2013. p. 732-735.

6. Wang $L, Z$ hang $Y, X u X$. A novel group detection method for finding related chinese herbs. Journal of Information Science \& Engineering. 2015;31:1387-411.

7. Ji W, Zhang $Y$, Wang $X$, Zhou Y. Latent semantic diagnosis in traditional chinese medicine. World Wide Web-internet \& Web Information Systems. 2017:20:1071-87.

8. Yao, L., Zhang, Y., Wei, B., Li, Z., Huang, X. Traditional Chinese medicine clinical records classification using knowledge-powered document embedding. In: 2016 IEEE International Conference on Bioinformatics and Biomedicine (BIBM) (2016), pp. 1926-1928.

9. He P, Deng K, Liu Z, Liu D, Liu JS, Geng Z. Discovering herbal functional groups of traditional chinese medicine. Statistics in Medicine. 2012;31:636-42.

10. Wu H, Zhang J. Analysis of prescription compatibility of headache external treatment of association rules in song dynasty. Journal of Emergency in Traditional Chinese Medicine. 2015;24:1365-6.

11. Liu T, Yu W, Fang Z, Kang J, Wang X. Prescription rules of professor yang muxiang in treatment of chronic gastritis based on asocciation rules and complex system entropy clustering. Chinese Journal of Experimental Traditional Medical Formulae. 2016;22:184-8.

12. Qin $\mathrm{Q}$, Tan $\mathrm{L}$. Top-rank-k frequent patterns mining algorithm based on tcm prescription database. Journal of Computer Applications. 2017;37:329-34. 
13. Han N, Qiao S, Gong X, Li T, Shu H, Yuan C. Mining compatibility of prescriptions over positive and negative association rules. Journal of Chinese Computer Systems. 2017;38:1538-42.

14. Liu K, Sun Y, Zhang D. An intelligent drug matching method for traditional chinese medicine; 2016. p. 150-154.

15. Weng H, He W, Ou A, Deng L, He C, Li H, Yan S. Ancient medical literature semantic annotation using hidden markov models. Proceedings - 2014 IEEE International Conference on Bioinformatics and Biomedicine, IEEE BIBM 2014; 2014. p. 37-40

16. Li S, Li Y, Li J, Wang M, Wang H, Yu X. Literature analysis on tcm syndromes and clinical characters of influenza. China Journal of Traditional Chinese Medicine \& Pharmacy. 2010;25:1286-9.

17. Xie D, Pei W, ZhuW, Li X. Traditional chinese medicine prescription mining based on abstract text; 2017. p. 1-5.

18. Zhou X, Liu B, Wu Z. Text mining for clinical chinese herbal medical knowledge discovery; 2005. p. 396-398.

19. Wang $Y, Y u Z$, Jiang $Y, X u K$, Chen $X$. Automatic symptom name normalization in clinical records of traditional chinese medicine. Bmc Bioinformatics. 2010;11:1-11.

20. Yao L, Mao ZJC, Zhang Y, Luo Y. Traditional chinese medicine clinical records classification with bert and domain specific corpora. Journal of the American Medical Informatics Association. 2019;26:1632-6.

21. Yao $L$, Zhang Y, Wei B, Zhang W, Jin Z. A topic modeling approach for traditional chinese medicine prescriptions. IEEE Transactions on Knowledge and Data Engineering. 2018;30:1007-21.
22. Dong F, Li X, Guo H, Tao O, Qiao Y. Study on prescription combination and design method based on dichotomy and greedy algorithm. China Journal of Chinese Materia Medica. 2014;39:2386-8.

23. Fu X, Song X, Wei L, Wang Z. Study of the distribution patterns of the constituent herbs in classical chinese medicine prescriptions treating respiratory disease by data mining methods. Chinese journal of integrative medicine. 2012;19:621-8.

24. Li S, Chen J, Lyu X. Analysis on composing prescriptions rules of prescriptions of coronary disease based on Ida topic model and association rules. Journal of New Chinese Medicine. 2018;50:47-50.

25. Wang L, Yin Z, Yun Z, Xu X, Cao S. Prescription function prediction using topic model and multilabel classifiers. Evid Based Complement Alternat Med. 2017;2017:1-10.

26. Ahmad Y, Ghovanlooy GF, Sima A, Somayeh H, Farshad M. Identifying association rules among drugs in prescription of a single drugstore using apriori method. Intelligent Information Management. 2015;7:253-9.

27. Wang M, Li J, Chen L, Huang Y, Zhou Q, Che L, Shang H. The study of the compatibility rules of traditional chinese medicine based on apriori and hmetis hypergraph partitioning algorithm. In: Biomedical Data Management and Graph Online Querying, pp. 16-31. Springer, Cham; 2016.

\section{Publisher's Note}

Springer Nature remains neutral with regard to jurisdictional claims in published maps and institutional affiliations.
Ready to submit your research? Choose BMC and benefit from:

- fast, convenient online submission

- thorough peer review by experienced researchers in your field

- rapid publication on acceptance

- support for research data, including large and complex data types

- gold Open Access which fosters wider collaboration and increased citations

- maximum visibility for your research: over $100 \mathrm{M}$ website views per year

At BMC, research is always in progress.

Learn more biomedcentral.com/submissions 